\title{
Correlation of E-cadherin Expression in Endometrial Carcinoma with Tumour Grade and Stage
}

\author{
Lizawati RH ${ }^{\mathrm{a}}$, Nur Maya Sabrina $\mathrm{TL}^{\mathrm{a}}$, Muhammad Fakhri MS ${ }^{\mathrm{a}}$, Azmawati MN ${ }^{\mathrm{b}}$, Nordashima $\mathrm{AS}^{\mathrm{a}}$ \\ ${ }^{a}$ Department of Pathology, Faculty of Medicine, Universiti Kebangsaan Malaysia Medical Centre, Kuala Lumpur, Malaysia. \\ ${ }^{b}$ Department of Community Health, Faculty of Medicine, Universiti Kebangsaan Malaysia Medical Centre, Malaysia
}

\begin{abstract}
Keywords

endometrial carcinoma, E-cadherin, tumour, gynaecology, pathology

Corresponding Author

Dr. Nordashima Abd. Shukor

Department of Pathology,

Faculty of Medicine,

Universiti Kebangsaan Malaysia,

Jalan Yaacob Latif,

Bandar Tun Razak,

56000 Cheras, Kuala Lumpur, Malaysia

No Tel: 0192217918

Email: nordashima@ppukm.ukm.edu.my

Received: 21 Dec 2020 ; Accepted: 6 April 2021

Doi: https://doi.org/10.31436/imjm.v20i3

INTRODUCTION: Endometrial carcinoma (EC) is among the common malignancy in the female with adverse prognosis in the advanced stage. Prediction of its prognosis is important in stratifying EC patients to achieve optimum treatment and improve clinical outcomes. This study is aimed to evaluate the prognostic significance of E-cadherin expression in patients with EC. The present study also investigated the correlation of Ecadherin expression in EC with its tumour grade and stage. MATERIALS AND METHODS: A total of 70 cases of EC were included in the study within eleven years comprising 56 cases of endometrioid carcinoma, 2 cases of mucinous carcinoma, 10 cases of serous carcinoma and 2 cases of clear cell carcinoma. E-cadherin expression was immunohistochemically analysed and compared with clinicopathological parameters. RESULTS: E-cadherin loss of expression shows significant association with nonendometrioid EC $(\mathrm{p}=0.003)$, high tumour grade $(\mathrm{p}<0.001)$ and tumour with distant metastasis $(p=0.028)$. Tumour grade is the main predictor of down-regulation of Ecadherin expression (Grade 3: aOR 8.400, 95\%CI 2.534-27.842). There was no significant association found between E-cadherin expression with myometrial invasion, FIGO stage, lymph node status and lymphovascular invasion. CONCLUSION: E-cadherin loss of expression correlates with poor prognostic factors namely high grade and high stage (metastasis) EC. This may serve as a potential prognostic marker for EC.
\end{abstract}

\section{INTRODUCTION}

Endometrial carcinoma (EC) is the second most common gynaecological malignancy worldwide with an estimate of 320000 new cases developing cancer annually and 76000 deaths in 2012. ${ }^{1}$ In Malaysia, EC was the seventh most common cancer in females. ${ }^{2}$

The widely accepted dualistic model for endometrial cancer which was proposed by Bockman had classified ECs to either type I or type II based on the endocrine and metabolic factors. ${ }^{3}$ Majority of tumours (approximately 80-90\%) are classified as type I EC which are generally low-grade endometrioid tumour, oestrogen-dependent carcinomas and behave less aggressively. Type II represents non-endometrioid endometrial carcinoma comprising of serous and clear cell carcinomas which are more aggressive with a poor prognosis.,5,6 The high grade (grade 3) endometrioid endometrial carcinomas, however, show overlap pathology with type II ECs. ${ }^{3}$ Many studies suggest aggressive tumour behaviour and poor survival rate for patients with serous and clear cell carcinoma are due to tumour propensity for extrauterine metastasis at the time of diagnosis. ${ }^{7,8}$

The disease progression depends on several factors including stage, depth of myometrial invasion, lymphovascular invasion, histological grade and nodal status.9 The overall 5-year survival rates for ECs are about $78-90 \%$ for stage I, 74\% for stage II, 36-57\% for stage III, and $20 \%$ for stage IV. ${ }^{10}$ In the majority of patients, surgical excision is the treatment of choice. However, adjuvant radiation therapy or chemotherapy (which is known to be associated with side effects) is considered for cases with residual disease or at higher 
risk of recurrence. Tailoring the optimum treatment for an individual patient is important to reduce unnecessary toxicity from adjuvant treatment. Therefore, finding better prognostic and predictive markers for EC is needed to guide the therapy and monitor the disease's progress for individual patients. ${ }^{11}$

The ongoing research in molecular pathways of EC has led to the identification of prognostic molecular markers that can detect patients with a higher risk of relapse or adverse outcome, among which E-cadherin is of interest. E-cadherin is an epithelial cell adhesion molecule consist of a transmembrane protein with a cytoplasmic domain that connects the actin cytoskeleton through a complex with its associated cytoplasmic proteins, $\alpha$-, $\beta$ - and $\gamma$-catenins. E-cadherin contributes to preserving tissue architecture. Previous studies revealed that reduced or absent E-cadherin expression is associated with invasiveness in vitro, facilitating metastasis in vivo and unfavourable clinicopathologic characteristics in several human cancers such as breast, ${ }^{12}$ stomach, ${ }^{13}$ and lung 14 as well as endometrial carcinoma. ${ }^{15-17}$ Down-regulation of E-cadherin plays a pivotal role in epithelial-to-mesenchymal transition (EMT), as well as alterations on other molecules involved in cell-cell adhesions, render cells with a migratory phenotype. ${ }^{18}$ One study has shown endometrial tumour with loss of E-cadherin expression is more likely to be poorly differentiated or type II endometrial tumour which often associated with poor prognosis. $^{7}$

Although the association between E-cadherin and EC are widely studied, to our knowledge, no published data is reporting on E-cadherin expression of $\mathrm{EC}$ in Malaysia. Thus, in this study, we investigated the expression of E-cadherin in endometrial carcinomas and its correlation with its tumour grade and stage.

\section{MATERIALS AND METHODS}

\section{Patients and Tissue samples}

This cross-sectional and descriptive study examined the histologic material, reports and medical record of all patients with endometrial carcinoma diagnosed in Universiti Kebangsaan Malaysia Medical Centre over eleven years and had undergone tumour resection. Clinicopathological parameters including age, ethnicity, histologic grade and its extend, lymphovascular invasion, metastases and depth of invasion were reviewed.

\section{Tissue microarrays (TMA)}

Tissue microarray (TMA) was constructed using Alphelys Minicore ${ }^{\circledR} 3$ Tissue Arrayer (Alphelys, Plaisir France). The tumour area was identified on H\&E and selected. Formalin-fixed, paraffin-embedded tissue blocks (donor) were retrieved and the region of interest was re-located to a recipient block. Double cores biopsied representative of $1.0 \mathrm{~mm}$ in diameter tissue core were taken from each tissue (donor) block and arrayed into empty paraffin blocks (recipient block) in a precisely spaced pattern.

\section{Immunohistochemistry (IHC)}

TMA blocks were sectioned approximately $3 \mu \mathrm{m}$ thickness and mounted on an adhesive glass slide. Primary antibody FLEX Monoclonal Mouse AntiHuman E-cadherin Clone NCH-38 Ready-to-Use (Code: IS059, Dako Denmark) was utilised in this study. The staining was performed using the protocol from EnVisionTM FLEX Mini Kit, High pH (Code No. K8023, Dako Denmark). The DAB Substrate Working Solution was made by diluting the $50 \mathrm{x}$ concentrated EnVisionTM FLEX DAB + Chromogen with EnvisionTM FLEX Substrate Buffer (Code No. K8023, Dako Denmark). The TMA sections were primarily incubated on the hot plate at $60^{\circ} \mathrm{C}$ for 1 hour. Next, the sections were dewaxed and pretreated within the Decloaking Chamber ${ }^{\mathrm{TM}}$ NxGen (Ref. No: DC2012220V, Biocare Medical California) using the EnVision ${ }^{\text {TM }}$ FLEX Target Retrieval Solution, High pH (Code No. DM828, Dako Denmark) at $110^{\circ} \mathrm{C}$ for 30 mins. Sections were then left to chill down at room temperature for 30 mins and rinsed under tap water for 3 mins. The tissue sections were then incubated with EnVisionTM FLEX Peroxidase-Blocking Reagent (Code No. DM821, Dako Denmark) for five mins followed by a washing step. TMA sections were then incubated with primary antibody for 30 mins at room temperature, followed by secondary antibody incubation with EnVisionTM FLEX/HRP (Code No. DM822, Dako Denmark) for 30 mins. Sections were then incubated with DAB Substrate 
Working Solution for five mins. After the procedures were finished, the slides were counterstained with Hematoxylin 2 (REF 7231, ThermoScientific USA) for five secs followed by a dehydration step with increasing alcohol solutions $(80 \%, 90 \%, 100 \%$ and 100\%) and 2 times Xylene. Finally, the slides were mounted using CoverSealTM-X xylene-based mounting medium (Cat. No.: FX2176, Cancer Diagnostics, USA). Breast carcinoma tissue was used as a positive control and included with each batch of TMA sections to confirm the consistency of the analysis.

\section{Immunohistochemical Analysis}

E-cadherin expression showed membranous staining and was scored semiquantitatively at 400 magnification following a scoring system developed by a previous study. ${ }^{17}$

E-cadherin expression was divided into two groups which were either the normal E-cadherin expression group or E-cadherin downregulation group. Tumour cells showing E-cadherin expression which was homogenous, strong membranous staining of more than $70 \%$ of tumour cells was classified under the normal E-cadherin expression group. E-cadherin expression which was completely lost or showing less than $70 \%$ of heterogenous, membranous staining, was considered as E-cadherin down-regulation group. ${ }^{17}$

\section{Statistical analysis}

Data analysis was performed using the Statistical Packaged for the Social Science (SPSS) software version 25.0. Pearson chi-square test and multiple logistic regressions were utilised to determine the association between different variables. A $p$-value of $\leq 0.05$ was considered statistically significant.

\section{RESULTS}

\section{Clinicopathological parameters}

A total of $70 \mathrm{EC}$ patients, who fulfilled the inclusion and exclusion criteria were included in this study. The youngest age at diagnosis was 32 years (ranged from 32 - 85 years, mean =61.4). Majority of cases were seen among Malay ethnic $(\mathrm{n}=38,54.3 \%)$ followed by
Chinese $(n=24,34.3 \%)$, Indian $(n=5,7.1 \%)$ and others $(n=3,4.2 \%)$. EC was more frequently seen in multiparous women $(n=53,75.7 \%)$ than nulliparous ( $n$ $=17,24.3 \%)$. The EC patients were mostly postmenopausal women with a frequency of $60(85.7 \%)$ and about 10 cases $(14.3 \%)$ were premenopausal women.

The majority of cases were endometrioid carcinomas (56/70, 80\%). The non-endometrioid carcinoma consisted of serous carcinoma (10/70, 14.3\%), mucinous carcinoma $(2 / 70,2.9 \%)$ and clear cell carcinoma $(2 / 70,2.9 \%)$. By using the FIGO grading system, the low-grade tumours comprised of 48 cases (68.6 \%) which include grade 1 (25/70, 35.7\%) and grade $2(23 / 70,32.9 \%)$ endometrioid and mucinous carcinoma. The high-grade tumours consist of grade 3 endometrioid carcinomas $(10 / 70,14.3 \%)$, serous carcinoma $(10 / 70,14.3 \%)$ and clear cell carcinoma $(2 / 70,2.9 \%)$.

Twenty-seven cases displayed tumour infiltration occupying less than $50 \%$ of the myometrial thickness (38.6\%) meanwhile 43 cases exhibited extensive myometrial infiltration of more than $50 \%$ of the myometrial thickness (61.4\%). A total of 42 cases $(60.0 \%)$ were staged as FIGO stage I, 8 cases $(11.4 \%)$ were at stage II disease, 13 cases $(18.6 \%)$ were at stage III and 7 cases $(10.0 \%)$ were categorised as stage IV.

There were only 11 cases $(15.7 \%)$ that showed the presence of lymphovascular invasion and lymph node involvement with 9 cases having distant metastasis $(12.9 \%)$.

\section{E-cadherin expression in Endometrial Carcinoma}

The correlations between E-cadherin expression in EC with clinicopathological parameters were summarised in Table I.

Down-regulation of E-cadherin expression was seen in more than half of the non-endometrioid tumours $(57.1 \%)$ in comparison to endometrioid tumours $(17.9 \%)$. There was a significant association between down-regulation of E-cadherin expression and histological type of tumour (endometrioid versus nonendometrioid) $(p=0.003)$. Similar findings were seen in tumour grade and distant metastases. There was a significant association between high tumour grade with 
down-regulation of E-cadherin expression $(\mathrm{p}<0.001)$. Down-regulation of E-cadherin expression was also seen in $55.6 \%$ of tumour with distant metastasis and $21.3 \%$ of tumour with an absence of distant metastasis. The correlation between down-regulation of E-cadherin expression and tumour with distant metastasis was statistically significant $(\mathrm{p}=0.028)$. However, there were no significant correlations found between E-cadherin expression with other clinicopathological factors, such as myometrial invasion, FIGO stage, lymphovascular invasion, and lymph node status (Table I).

TABLE I: Correlation between E-cadherin expression and clinicopathological parameters

\begin{tabular}{|c|c|c|c|c|}
\hline \multicolumn{2}{|c|}{ Parameters } & \multicolumn{3}{|c|}{$\begin{array}{l}\text { Number of cases (\%) with } \\
\text { E-cadherin expression }\end{array}$} \\
\hline \multirow{3}{*}{$\begin{array}{l}\text { Tumour } \\
\text { type }\end{array}$} & & $\begin{array}{c}\text { Down- } \\
\text { regulation }\end{array}$ & Normal & $\mathrm{p}$-value \\
\hline & Endometrioid & $10(17.9)$ & $46(82.1)$ & 0.003 \\
\hline & $\begin{array}{l}\text { Non- } \\
\text { endometrioid }\end{array}$ & $8(57.1)$ & $6(42.9)$ & \\
\hline \multirow{2}{*}{$\begin{array}{l}\text { Tumour } \\
\text { grade }\end{array}$} & Low & $6(12.5)$ & $42(87.5)$ & $<0.001$ \\
\hline & High & $12(54.5)$ & $10(45.5)$ & \\
\hline \multirow{2}{*}{$\begin{array}{l}\text { Myometrial } \\
\text { invasion }\end{array}$} & $<1 / 2$ & $6(22.2)$ & $21(77.8)$ & 0.596 \\
\hline & $\geq 1 / 2$ & $12(27.9)$ & $31(72.1)$ & \\
\hline \multirow{2}{*}{$\begin{array}{l}\text { FIGO } \\
\text { stage }\end{array}$} & $1-2$ & $11(22.0)$ & $39(78.0)$ & 0.261 \\
\hline & $3-4$ & $7(35.0)$ & $13(65.0)$ & \\
\hline \multirow{2}{*}{$\begin{array}{l}\text { Lympho- } \\
\text { vascular } \\
\text { invasion }\end{array}$} & Yes & $5(45.5)$ & $6(54.5)$ & 0.103 \\
\hline & No & $13(22.0)$ & $46(78.0)$ & \\
\hline \multirow{2}{*}{$\begin{array}{l}\text { Lymph } \\
\text { node } \\
\text { metastasis }\end{array}$} & Yes & $4(36.4)$ & $7(63.6)$ & 0.379 \\
\hline & No & $14(23.7)$ & $45(76.3)$ & \\
\hline \multirow{2}{*}{$\begin{array}{l}\text { Distant } \\
\text { metastasis }\end{array}$} & Positive & $5(55.6)$ & $4(44.4)$ & 0.028 \\
\hline & Negative & $13(21.3)$ & 48 (78.7) & \\
\hline
\end{tabular}

Univariable logistic regression found tumour histological type, grade and distant metastasis to be significant predictors of E-cadherin expression (Table II). After controlling the confounder of other variables in multivariable analysis, the only significant predictor of E-cadherin expression is the tumour grade $(\mathrm{p}=<0.001$; odds ratio $[\mathrm{OR}] \mathrm{8.4} ; 95 \%$ confidence interval [CI] 2.534-27.842) (Table III).

\section{DISCUSSION}

E-cadherin is recognised as the main component in cellcell adhesion and reduced/loss of E-cadherin expression is recognised as part of the molecular event that leads to cancer invasion and metastasis. ${ }^{19}$ The MJM Volume 20 No.3, July 2021
TABLE II: Single logistic regression

\begin{tabular}{|c|c|c|c|c|c|c|}
\hline \multicolumn{2}{|c|}{ Variable } & \multirow[t]{2}{*}{$\mathbf{B}$} & \multirow[t]{2}{*}{ aOR } & \multicolumn{2}{|c|}{$95 \% \mathrm{CI}$} & \multirow{2}{*}{$\begin{array}{l}\mathrm{p}- \\
\text { value }\end{array}$} \\
\hline & & & & Lower & Upper & \\
\hline \multirow{2}{*}{$\begin{array}{l}\text { Tumour } \\
\text { type }\end{array}$} & Endometrioid & \multicolumn{4}{|c|}{ Reference } & \\
\hline & $\begin{array}{l}\text { Non- } \\
\text { endometrioid }\end{array}$ & 1.814 & 6.133 & 1.739 & 21.627 & 0.005 \\
\hline \multirow{2}{*}{$\begin{array}{l}\text { Tumour } \\
\text { grade }\end{array}$} & Low grade & \multicolumn{4}{|c|}{ Reference } & \\
\hline & High grade & 2.128 & 8.400 & 2.534 & 27.842 & $<0.001$ \\
\hline \multirow{2}{*}{$\begin{array}{l}\text { Distant } \\
\text { metastasis }\end{array}$} & Negative & \multicolumn{4}{|c|}{ Reference } & \\
\hline & Positive & 1.529 & 4.615 & 1.082 & 19.687 & 0.039 \\
\hline
\end{tabular}

present study revealed that down-regulation of E-cadherin expression is significantly associated with non-endometrioid carcinoma, higher tumour grade and stage. Our results showed that loss of E-cadherin expression is significantly correlated with nonendometrioid carcinoma which is consistent with the previous studies ${ }^{7}$ which concluded that it was likely due to biological aggressiveness in the non-endometrioid tumour.

TABLE III: Multiple logistic regression

\begin{tabular}{|c|c|c|c|c|c|c|}
\hline \multicolumn{2}{|c|}{ Variable } & \multirow[t]{2}{*}{$\bar{B}$} & \multirow[t]{2}{*}{ aOR } & \multicolumn{2}{|l|}{$95 \% \mathrm{CI}$} & \multirow[t]{2}{*}{ p-value } \\
\hline \multirow{3}{*}{$\begin{array}{l}\text { Tumour } \\
\text { grade }\end{array}$} & & & & Lower & Upper & \\
\hline & $\begin{array}{l}\text { Low } \\
\text { grade }\end{array}$ & \multicolumn{4}{|c|}{ Reference } & \\
\hline & $\begin{array}{l}\text { High } \\
\text { grade }\end{array}$ & 2.128 & 8.400 & 2.534 & 27.842 & $<0.001$ \\
\hline
\end{tabular}

The histological grade of a tumour remains one of the most important prognostic factors in EC. Most of our cases were low-grade tumour (grade 1 and 2) that behaviorally is less aggressive and associated with better outcome and prognosis. In this study, we found that decreased E-cadherin expression is associated with higher grade EC (54.5\%) compared to lowgrade EC (12.5\%). Normal expression was significantly observed in low grade (grade 1 and 2) tumour while decreased expression was observed in grade 3 tumour. A similar finding is seen in the first study to report the relationship between $\mathrm{E}$ cadherin expression and grade of the tumour, myometrial invasion and lymph node metastasis by Sakuragi et al. ${ }^{20}$ This finding is parallel with some other previous studies by Sugihara, ${ }^{17}$ Florescu et al, ${ }^{21}$ Holcomb et al, ${ }^{7}$ Yalta et al, ${ }^{22}$ Ahmed and Muhammad. ${ }^{23}$ These previous studies concluded that E-cadherin expression was stronger in well-differentiated tumours. Our data is in line with these observations. This can be concluded that decreased expression may account for the aggressiveness of the higher-grade tumour. ${ }^{22}$ Shaco-Levy et al ${ }^{24}$ noted the opposite finding where they noticed that significant loss of E-cadherin was also observed in low grade endometrioid endometrial carcinoma where the authors studied 39 cases comprised of 15 grade 1 endometrioid carcinoma, 14 serous carcinoma and 10 cases of proliferative endometrium. 


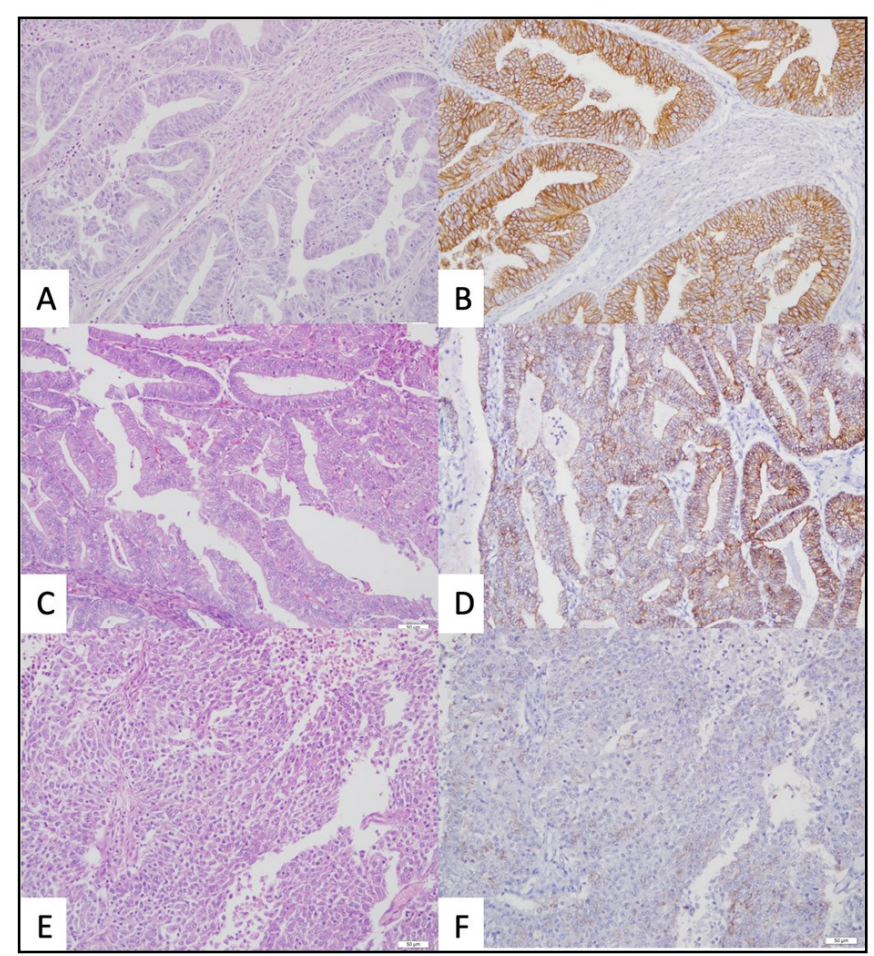

FIGURE 1: Endometrial carcinoma (H\&E and E-cadherin immunohistochemistry, 200x magnification) (A) Endometrioid carcinoma, grade 1 (B) with homogenous E-cadherin expression (C) Endometrioid carcinoma, grade 3 (D) with heterogenous E-cadherin expression (E) Serous carcinoma, grade 3 (F) with negative Ecadherin expression.

Our data suggest that loss of E-cadherin expression was a significant predictor for high tumour grade and remained as an independent predictor when conducted the multivariate analysis. This observation is similarly seen in a study by Sakuragi et al. ${ }^{20}$

It is interesting to note in our study, although Ecadherin expression is one of the important prognostic markers, the expression is variable in previous studies. This study showed $18(75 \%)$ cases out of 70 EC loss E-cadherin expressions. Previous studies stated percentages of decreased or negative expression in EC vary from $11 \%$ to $60 \% .25$ Several possible reasons were identified which contribute to this heterogeneity and one of them was due to the relatively small sample size in most series. Noteworthy, the use of the different scoring system and lack of standardisation is a major inherent problem when comparing results across different studies. To date, there is no standard guideline for E-cadherin testing established for EC other than it is primarily being used to assist in the diagnosis of lobular carcinoma of the breast. We follow the latest study done by Sugihara ${ }^{17}$ which in our opinion shows a more relevant scoring system by interpreting both heterogeneous and negative E-cadherin staining as E- cadherin down-regulation group and homogenous Ecadherin expression as normal E-cadherin expression group. However, this scoring system needs further validation to ensure reliable and reproducible study outcomes.

Our findings showed a tumour with distant metastasis $(55.6 \%)$ were more likely to have a loss of E-cadherin expression and this finding was statistically significant. A study by Loh et al and Tanaka et al showed that epithelial-to-mesenchymal transition (EMT) was essential in tumorigenesis where the EMT program enhances metastasis, chemoresistance and tumour stemness.26,27 The hallmark of EMT is the upregulation of N-cadherin followed by the downregulation of Ecadherin which is regulated by a complex network of signalling pathways and transcription factors. 26,27

In our study, $71.4 \%$ of the cases were diagnosed in the early stage (stage I and II) and $28.6 \%$ of cases were in stage III and IV. Our study did not show a significant correlation with E-cadherin expression. We also observed that even though tumour with a higher FIGO stage is more likely to have decreased E-cadherin expression, the difference was not statistically significant. A similar observation was seen in a study by Moreno-Bueno et $\mathrm{al}^{16}$ in a large sample that they studied comprised of 149 endometrial carcinomas including 21 atypical endometrial hyperplasias. They found out that $85.7 \%$ of endometrial carcinoma with stage III-IV showed significant loss of E-cadherin expression. This has also been shown in a study by Holcomb et al.?

Similar findings were seen in tumour invasion of the myometrium. Our study showed that there was no significant association between E-cadherin expression and the depth of the myometrial invasion. On contrary, other studies found that tumour seemed to invade deeper into the myometrium when the tumours had lost E-cadherin expression. 7,20 However another study has shown that decreased E-cadherin expression was found to be correlated with decreased myometrial invasion. ${ }^{28}$ This may reflect a complex biological process such as stromal invasion which is not only regulated by a single molecular alteration, rather by a sequential change involving alteration of several adhesion molecules that might play an important role in tumour progression ${ }^{25}$. 
Although we detected there is a tendency for tumour with lymphovascular invasion and lymph node metastasis to have a loss of E-cadherin expression, nevertheless the relationship was not statistically significant. The statistical insignificance in our study was possibly related to a smaller number of patients recruited or possible sampling limitation.

\section{CONCLUSION}

Our study revealed that loss of E-cadherin expression is significantly associated with non-endometrioid carcinomas, higher tumour grade and was found to be more common in tumour with distant metastasis. This study showed that E-cadherin may serve as a potential prognostic marker in EC and provide additional information in predicting tumour behaviour and may aid in the selection of patients for more aggressive adjuvant therapy. A follow-up study with a larger number of samples and further research correlating its molecular basis is needed to confirm the usefulness of E-cadherin.

\section{ACKNOWLEDGEMENT}

This study was supported by UKMMC Fundamental Research Fund (UKM FPR.4/244/FF-2018-100).

\section{CONFLICT OF INTEREST}

None

\section{REFERENCES}

1. Ferlay J, Soerjomataram I, Dikshit R, et al. Cancer incidence and mortality worldwide: sources, methods and major patterns in GLOBOCAN 2012. Int J Cancer 2015;136:E359-86.

2. Azizah Ab M, Nor Saleha I.T, Noor Hashimah A, Asmah Z.A, W M. MALAYSIAN NATIONAL CANCER REGISTRY REPORT 2007-2011. Putrajaya, Malaysia: The National Cancer Institute, Ministry of Health Malaysia; 2016 June 2015.

3. Buhtoiarova TN, Brenner CA, Singh M.

Endometrial Carcinoma: Role of Current and Emerging Biomarkers in Resolving Persistent
Clinical Dilemmas. American journal of clinical pathology 2016;145:8-21.

4. Passarello K, Kurian S, Villanueva V. Endometrial Cancer: An Overview of Pathophysiology, Management, and Care. Seminars in oncology nursing 2019;35:157-65.

5. Park JY, Nam JH, Kim YT, et al. Poor prognosis of uterine serous carcinoma compared with grade 3 endometrioid carcinoma in early stage patients. Virchows Archiv : an international journal of pathology 2013;462:289-96.

6. Hamilton CA, Cheung MK, Osann K, et al. Uterine papillary serous and clear cell carcinomas predict for poorer survival compared to grade 3 endometrioid corpus cancers. British journal of cancer 2006;94:642-6.

7. Holcomb K, Delatorre R, Pedemonte B, McLeod C, Anderson L, Chambers J. E-cadherin expression in endometrioid, papillary serous, and clear cell carcinoma of the endometrium. Obstetrics and gynecology 2002;100:1290-5.

8. Alkushi A, Kobel M, Kalloger SE, Gilks CB. Highgrade endometrial carcinoma: serous and grade 3 endometrioid carcinomas have different immunophenotypes and outcomes. International journal of gynecological pathology : official journal of the International Society of Gynecological Pathologists 2010;29:343-50.

9. Edge SB, Compton CC. The American Joint Committee on Cancer: the 7th edition of the AJCC cancer staging manual and the future of TNM. Ann Surg Oncol 2010;17:1471-4.

10. Lewin SN, Herzog TJ, Barrena Medel NI, et al. Comparative performance of the 2009 international Federation of gynecology and obstetrics' staging system for uterine corpus cancer. Obstetrics and gynecology 2010;116:1141-9.

11. Hutt S, Tailor A, Ellis P, Michael A, Butler-Manuel $\mathrm{S}$, Chatterjee J. The role of biomarkers in endometrial cancer and hyperplasia: a literature review. Acta oncologica 2019;58:342-52.

12. Siitonen SM, Kononen JT, Helin HJ, Rantala IS, Holli KA, Isola JJ. Reduced E-cadherin expression is associated with invasiveness and unfavorable prognosis in breast cancer. American journal of clinical pathology 1996;105:394-402. 
13. Gabbert HE, Mueller W, Schneiders A, et al. Prognostic value of E-cadherin expression in 413 gastric carcinomas. Int J Cancer 1996;69:184-9.

14. Bremnes RM, Veve R, Gabrielson E, et al. Highthroughput tissue microarray analysis used to evaluate biology and prognostic significance of the E-cadherin pathway in non-small-cell lung cancer. J Clin Oncol 2002;20:2417-28.

15. Leblanc M, Poncelet C, Soriano D, et al. Alteration of CD44 and cadherins expression: possible association with augmented aggressiveness and invasiveness of endometrial carcinoma. Virchows Archiv : an international journal of pathology 2001;438:78-85.

16. Moreno-Bueno G, Hardisson D, Sarrio D, et al. Abnormalities of E- and P-cadherin and catenin (beta-, gamma-catenin, and p120ctn) expression in endometrial cancer and endometrial atypical hyperplasia. J Pathol 2003;199:471-8.

17. Sugihara T. Loss of Adherens Junction Protein ECadherin is a Biomarker of High-Grade Histology and Poor Prognosis in Endometrial Cancer. Ann Clin Lab Res 2016;4:1.

18. Abal M, Llaurado M, Doll A, et al. Molecular determinants of invasion in endometrial cancer. Clinical \& translational oncology : official publication of the Federation of Spanish Oncology Societies and of the National Cancer Institute of Mexico 2007;9:272-7.

19. Mansour M, Teo ZL, Luen SJ, Loi S. Advancing Immunotherapy in Metastatic Breast Cancer. Current treatment options in oncology 2017;18:35.

20. Sakuragi N, Nishiya M, Ikeda K, et al. Decreased Ecadherin expression in endometrial carcinoma is associated with tumor dedifferentiation and deep myometrial invasion. Gynecol Oncol 1994;53:1839.

21. Florescu MM, Pirici D, Simionescu CE, et al. Ecadherin and beta-catenin immunoexpression in endometrioid endometrial carcinoma. Rom J Morphol Embryol 2016;57:1235-40.

22. Yalta T, Atay L, Atalay F, Caydere M, Gonultas M, Ustun H. E-cadherin expression in endometrial malignancies: comparison between endometrioid and non-endometrioid carcinomas. J Int Med Res 2009;37:163-8.
23. Ahmed AR, Muhammad EM. E-cadherin and CD10 expression in atypical hyperplastic and malignant endometrial lesions. J Egypt Natl Canc Inst 2014;26:211-7.

24. Shaco-Levy R, Sharabi S, Benharroch D, Piura B, Sion-Vardy N. Matrix metalloproteinases 2 and 9, E-cadherin, and beta-catenin expression in endometriosis, low-grade endometrial carcinoma and non-neoplastic eutopic endometrium. European journal of obstetrics, gynecology, and reproductive biology 2008;139:226-32.

25. Scholten AN, Aliredjo R, Creutzberg CL, Smit VT. Combined E-cadherin, alpha-catenin, and betacatenin expression is a favorable prognostic factor in endometrial carcinoma. Int J Gynecol Cancer 2006;16:1379-85.

26. Loh CY, Chai JY, Tang TF, et al. The E-Cadherin and N-Cadherin Switch in Epithelial-toMesenchymal Transition: Signaling, Therapeutic Implications, and Challenges. Cells 2019;8.

27. Tanaka Y, Terai Y, Kawaguchi H, et al. Prognostic impact of EMT (epithelial-mesenchymaltransition)-related protein expression in endometrial cancer. Cancer biology \& therapy 2013;14:13-9.

28. Mell LK, Meyer JJ, Tretiakova M, et al. Prognostic significance of E-cadherin protein expression in pathological stage I-III endometrial cancer. Clin Cancer Res 2004;10:5546-53. 